\title{
Production, safety, health effects and applications of diacylglycerol functional oil in food systems: a review
}

\begin{abstract}
Diacylglycerol (DAG) is a world leading anti-obesity functional cooking oil synthesized via structural modification of conventional fats and oils. DAG exits in three stereoisomers namely sn-1,2-DAG, sn-1,3-DAG, and sn-2,3-DAG. DAG particularly sn-1,3-DAG demonstrated to have the potential in suppressing body fat accumulation and lowering postprandial serum triacylglycerol, cholesterol and glucose level. DAG also showed to improve bone health. This is attributed to DAG structure itself that caused it to absorb and digest via different metabolic pathway than conventional fats and oils. With its purported health benefits, many studies attempt to enzymatically or chemically synthesis DAG through various routes. DAG has also received wide attention as low calorie fat substitute and has been incorporated into various food matrixes. Despite being claimed as healthy cooking oil the safety of DAG still remained uncertain. DAG was banned from sale as it was found to contain probable carcinogen glycidol fatty acid esters. The article aims to provide a comprehensive and latest review of DAG emphasizing on its structure and properties, safety and regulation, process developments, metabolism and beneficial health attributes as well as its applications in the food industry.
\end{abstract}

Keyword: Diacylglycerol; Enzyme lipase; Obesity; Emulsion; Glycidol ester; Production 